\title{
Raza y género en el marco de la diferencia ontológica colonial
}

Race and gender in the colonial ontological difference framework

\author{
Jhon Jairo losada Cubillos \\ Universidad Católica de Lovaina, Bélgica
}

\begin{abstract}
RESUMEN En los últimos años el pensamiento decolonial se ha constituido en un campo de reflexión teórica y de activismo político que busca, por un lado, visibilizar las formas en las que se sigue reproduciendo el poder colonial y, por otro lado, proveer herramientas para contribuir a fisurar las condiciones de dominación heredadas del hecho colonial. Este artículo tiene por objetivo situar las categorías de raza y género en el marco del pensamiento decolonial, como categorías fundamentales para problematizar y analizar la operacionalidad la dimensión ontológica de la colonialidad. En este sentido, recurre a autores y autoras de la tradición de pensamiento crítico afrocaribeño, así como del feminismo decolonial. Para esto, se discute con la categoría de diferencia ontológica colonial, como un aporte para continuar exteriorizando la naturaleza del orden colonial de poder y para ahondar en la comprensión en la forma cómo la colonialidad opera.
\end{abstract}

PALABRAS CLAVE Colonialidad; género; raza; diferencial ontológica colonial.

ABSTRACT In recent years, decolonial thinking has become a field of theoretical reflection and political activism that seeks, on the one hand, to make visible the ways in which the colonial power continues to be reproduced, and, on the other hand, to provide tools to contribute to fissure the conditions of domination inherited from the colonial fact. This article aims to place the categories of race and gender, in the framework of decolonial thought, as fundamental categories to problematize and analyze the ontological dimension of coloniality. In this regard, it draws on authors from the Afro-Caribbean critical thinking tradition, as well as from decolonial feminism. For this, a dialogue with the 
category of colonial ontological difference is created, as a contribution to continue externalizing the nature of the colonial order of power and to deepen the understanding of how coloniality operates.

KEYWORDS Coloniality; gender; race; colonial ontological difference.

\section{Introducción}

La reflexión que se presenta a continuación se inserta en el marco de los estudios decoloniales en América Latina. En este sentido, y atendiendo al llamado de hacer visibles los mecanismos de poder que reproducen en la actualidad el legado colonial en contextos que cargaron ("cargan") con el yugo colonialista, este trabajo se propone como una contribución crítica para entender las dinámicas de la operacionalidad de la colonialidad, así como proveer elementos para dar pasos hacia el camino a la decolonización.

Para este caso en particular, el presente artículo plantea una discusión en torno a las ideas de raza y género como agentes centrales de lo que aquí se reconoce como la "dimensión ontológica de la colonialidad". Con esto, se busca marcar un campo diferencial a nivel conceptual y metodológico del campo discursivo, en el que el poder colonial debe ser reconocido. De esta forma, la pregunta por el qué (la naturaleza) y el cómo (formas de operación) de la colonialidad se hacen vigentes en tanto que se continúan legitimando mecanismos de poder que persisten en la actualidad.

En este documento se torna de especial relevancia asumir el cuestionamiento alrededor de la forma cómo la colonialidad continúa reproduciendo diversas formas de exclusión, desigualdades sociales y permanentes relaciones de dominación, se tornan de especial relevancia. Para este caso, se propone analizar las ideas de raza y género como las bases sobre las cuales se funda, se constituye la colonialidad para desplegar su potencial hegemónico. Escenarios justamente como la formación de subjetividades, del sentido común, espacios de intimidad, visiones de mundo, entre otros harían parte de lo que se plantea como la "dimensión ontológica" en tanto que forman y dan sentido a la experiencia vivida misma del sujeto colonial.

En otras palabras, la presente exposición más que dirigirse a una lectura de aspectos discursivos que indague por ejemplo, por la "sexualidad" o la "condición racional" (o diversas identidades coloniales) como regímenes de representación a partir de los cuales, se analiza la naturaleza de la relación entre sexualidad y poder, violencia y género, raza e identidad, la legitimidad o no de las luchas por el derecho de las mujeres, afrodescendientes e incluso de indígenas, las formas de violencia en la que se da lo masculino y lo femenino en contextos coloniales, etc.; se propone más bien una lectura en clave ontológica de elementos como la raza y el género y las prácticas que en torno a estos se fundan y que dinamizan el poder colonial en la vida, en el espacio 
circundante del sujeto colonizado, a partir de lo cual se justifica la subordinación, la violencia física, ataques racistas, feminicidios, etc.

Con esto se pretende acentuar dos elementos importantes para la comprensión del siguiente artículo: por un lado, la necesidad de avanzar en la comprensión de la ontología del poder colonial, para continuar afinando la comprensión de la forma cómo la colonialidad. En este sentido, se sostiene que el poder colonial funciona legitimando las prácticas sexistas y racistas a partir de lo que, autores como Maldonado-Torres (2007) reconocen como diferencia ontológica colonial. De esta forma, se entiende a la diferencia ontológica colonial como una estrategia de poder que encubre e invisibiliza niveles de violencia, jerarquías y estructuras de dominación que reproducen a su vez el legado colonial.

Por otro lado, el segundo elemento fundamental de la siguiente disertación, tiene que ver con el hecho de avanzar en la comprensión de la matriz ontológica del poder colonial más allá de su lectura discursiva (que se ocupa de aspectos diferentes), abre posibilidades para la decolonización y la lucha emancipatoria. De no hacerlo, es decir, de no dar este paso en la compresión del poder colonial, el esfuerzo por avanzar hacia la decolonización o cualquier intento de desbordar la colonialidad, fisurarla y criticarla no solo será vano, sino además se verá comprometido en seguir el juego que ésta propone, en continuar con la reproducción hegemónica de las estructuras coloniales de poder.

\section{Sobre la diferencia ontológica colonial como proyecto de muerte}

La primera tesis que se propone sobre la operatividad de la colonialidad, es que ésta se despliega de la misma manera al nivel de la formación de la idea de género como la de la raza. De esta forma, se advierte que tanto las prácticas racistas como las sexistas, se encuentran emplazadas en la misma matriz de poder colonial, producto del anclaje de la diferencia ontológica colonial en el mundo circundante del sujeto colonizado.

La preocupación por pensar la dimensión ontológica de la colonialidad, de acuerdo a los intereses investigativos desde los cuales se deriva el presente trabajo, tiene que ver, por un lado, con el hecho de considerar un campo, hasta ahora poco estudiado, de operatividad del poder colonial que se manifiesta en la experiencia vivida misma de los sujetos colonizados. Por otro lado, por indagar en torno a la necesidad de avanzar hacia un régimen político, no meramente discursivo, que permita construir formas de ser y estar en el mundo más allá de las condiciones dominación colonial.

Desde la perspectiva decolonial se asumen estas condiciones en el marco de un proyecto hegemónico-ideológico y por ello mismo ontológico que emerge a partir de 1492, con el encubrimiento de "América" y, a su vez, el nacimiento del sistema-mundo-moderno-colonial-capitalista. Sobre esta idea del nacimiento del sistema-mundo, Quijano y Wallerstein señalaran enfáticamente que: "El moderno sistema mundial 
nació a lo largo del siglo XVI. América -como entidad geosocial- nació a lo largo del siglo XVI. La creación de esta entidad geosocial, América, fue el acto constitutivo del moderno sistema mundial" (Quijano \& Wallerstein, 1992, p. 583).

Lo señalado por estos autores permite evidenciar la necesidad de desnaturalizar esta matriz constituyente (dadora de sentido), que ha hecho que el poder colonial se ancle, es decir, se acentúe en la dimensión más íntima del sujeto colonizado, a saber, la experiencia vivida misma en tanto cosa colonizada. En sintonía a lo anterior y de la mano de Dussel se puede agregar lo siguiente:

Para ello es necesario destituir al ser de su pretendida fundamentalidad eterna y divina; negar la religión fetichista; mostrar a la ontología como la ideología de las ideologías; desenmascarar los funcionalismos, sean estructuralistas, lógico-cientificistas o matematizantes, que al pretender que la razón no puede criticar dialécticamente el todo, lo afirma por más analíticamente que critiquen u operativicen sus partes (Dussel, 1996, p. 18).

Así las cosas, se reconoce por matriz colonial o colonialidad al aparato de dominación que emerge en 1492 a partir de lo que Dussel ha denominado el "encubrimiento de América" (Dussel, 1992) y que se constituye en la cara oculta o lado oscuro de la modernidad (Mignolo, 1995). Adicionalmente, la colonialidad se entiende como un fenómeno complejo y de larga duración que se refiere a un patrón de poder que opera a través de la naturalización de las jerarquías a nivel del territorio, de la cultura, de los sentidos, de los conocimientos, del sexo, la clase, la raza, etc. que posibilitan y dinamizan de manera permanente relaciones de dominación.

Así mismo, se puede destacar que este poder opera en dos dimensiones. El nivel discursivo de la colonialidad se sustenta bajo la idea de diferencia colonial. A partir de esta categoría y claramente en sintonía con los aportes de Michel Foucault se identifican los mecanismos de poder (dispositivos) de orden "discursivo" (poder-saber), utilizados para la dinamización y despliegue la colonialidad como estrategia de dominación, diferente al poder político y militar del colonialismo. En otras palabras, con esto se hace referencia a los imaginarios culturales, representaciones simbólicas y criterios epistemológicos que recrean o reproducen el poder colonial a partir de la idea de la diferencia colonial (por ejemplo, la constitución misma de la idea de América Latina, como lo ha mostrado Mignolo, 2005).

En este nivel discursivo se reconoce la colonialidad del poder, con trabajos como los de Quijano (1992; 2000a; 2000b; 2005; 2007), Mignolo (2000; 2005), Escobar (1996; 1999), Grosfoguel (2003; 2005; 2006), entre otros; así mismo, se pone de manifiesto la colonialidad del saber, con un amplio desarrollo por parte de autores como Lander (2005); Castro-Gómez (1996; 2005; 2007; 2009); Dussel (1992; 1999; 2005), etc.

Ahora bien, si el nivel discursivo de la colonialidad (poder-saber) se sustenta bajo la idea de la "diferencia colonial", el nivel ontológico haya su anclaje en la "diferencia 
ontológica colonial". Con esto se entiende a la colonialidad como un poder constituyente, divisorio y antagónico (civilización-barbarie-desarrollo-subdesarrollo, etc.), que no se reduce ni se limita al nivel óntico, es decir, al terreno de las cosas particulares. En ese sentido, va mucho más allá de las tasas de pobreza, de analfabetismo, de desnutrición o de desempleo; tampoco se agota meramente en la construcción de subjetividades, identidades, regímenes de representación cultural (pobres, subdesarrollados, excluidos, etc.), como se anota desde el nivel discursivo de la decolonialidad; sino que genera un panorama de violencia (racial, sexista, etc.), que ejerce su dominio invisibilizando su misma operación, justamente es lo que considero como la “dimensión ontológica de la colonialidad”. Esta gramática de poder no es localizable empíricamente, es constituyente y en ese sentido opera en el plano del ser (experiencia racial del sujeto colonizado) y del género (relaciones patriarcales).

En este sentido, la lectura ontológica del poder colonial es importante y necesaria porque éste opera y tiene éxito gracias a que oculta su propia naturalización de verdades ontológicas, además establece prácticas políticas para mantener su poderío; esto es lo que se reconoce como "mala fe" (Gordon, 1999, Suárez-Krabbe, 2016). Por otra parte, hay que añadir que los avances en el nivel ontológico de la colonialidad (colonialidad del ser, colonialidad del género), no han sido tan numerosos y es justamente en este escenario en el que se inserta el presente documento. En este orden podemos encontrar los trabajos de Maldonado-Torres (2003, 2006; 2007; etc.), Wynter (2004) Lugones $(2008,2011)$, entre otros autores y autoras que han dado pistas relevantes para adentrarnos en este dominio.

Cuando señalamos la naturalización y legitimidad del poder colonial, en tanto acto de "Mala fe", y la relacionamos con el ámbito ontológico nos referimos al ser de los sujetos colonizados (deshumanizados), independientemente sea este hombre o mujer. Con esto queremos acentuar que nos referimos a la dimensión más íntima del ser humano y a las interacciones íntimas entre estos. Es allí donde se localiza y encuentra su posicionamiento la colonialidad del género. Ciertamente esta zona de intimidad ha sido uno de los aspectos fuertes del trabajo de María Lugones. Sobre esto, Lugones afirma: "Cuando pienso en intimidad aquí, no estoy pensando exclusivamente ni principalmente sobre relaciones sexuales. Estoy pensando en la vida social entretejida entre personas que no están actuando como representantes o funcionarias" (Lugones, 2011, p. 107).

Situarse desde esta zona de intimidad lleva a visualizar la acción de restricción y despojo de la vida, lo cual significa, en el sentido más amplio, la muerte. Sobre esto, afirma Lugones:

Al usar el término colonialidad mi intención es nombrar no sólo una clasificación de pueblos en términos de la colonialidad de poder y el género, sino también el proceso de reducción activa de las personas, la deshumani- 
zación que los hace aptos para la clasificación, el proceso de subjetificación, el intento de convertir a los colonizados en menos que seres humanos (Lugones, 2011, p. 108).

Dicho proceso de violencia, de reducción activa de las personas, de deshumanización y subjetificación (Lugones, 2011), de Mala fe (Gordon, 1999; Suárez-Krabbe, 2016) que permea en la zona más íntima del ser es ciertamente una de las consecuencias de la constitución de la diferencia ontológica colonial al clasificarlos, ordenarlos, asignarles identidades y roles dentro de la sociedad como "lugares naturales", incluso restringiendo la vida y generando muerte.

Según Maldonado-Torres (2007) este ámbito ontológico de la colonialidad responde a la necesidad de aclarar la pregunta sobre los efectos de la colonialidad en la experiencia vivida, y no sólo en la mente de sujetos subalternos, es decir, determinar las formas mediante las cuales se naturaliza e interioriza la diferencia ontológica colonial. A partir de esta categoría, se interroga por el ser de los seres que han sido encubiertos y dotados de una condición sub-ontológica que los sitúa jerárquicamente por debajo de otros seres gracias a la dinámica colonial, instaurando así una suerte de escepticismo sobre la humanidad de los "sub-otros colonizados" (Maldonado-Torres, 2007). Se trata entonces de la interiorización de una condición sub-ontológica, una relación jerárquica entre el ser y lo que está más debajo de éste, una conexión entre ontología y el poder colonial.

Sin embargo, más allá de esto con la diferencia ontológica colonial se establece aquello que se encuentra en la línea del ser y aquello que está por debajo de la misma (nivel sub-ontológico $)^{1}$. Por ello, se justifica la recurrencia a la violencia para amedrentarlo, inferiorizarlo y subordinarlo en un proyecto de muerte. En tanto proyecto, se materializa a través de esquemas e instituciones coloniales que se forman a partir de ficciones ontológicas y que legitiman las desigualdades y que hacen viva la colonialidad, gracias a que se encargan de ocultar las verdades que sostienen estas estructuras, y de recrear los mecanismos políticos que garantizan su reproducción. Para Suárez-Krabbe (2014; 2016), este proyecto de muerte apunta al conjunto de prácticas hegemónicas, donde están íntimamente ligados el capitalismo, el patriarcado, la colonialidad, el racismo y las prácticas depredadoras en contra de la naturaleza.

Así las cosas, el proyecto de muerte es ante todo un proyecto ontológico que defiende una segregación global, en la cual se protege la vida, los privilegios y la riqueza de unos pocos a costa de la vida de la gran mayoría de la población mundial. Este

1. Recordamos el famoso pasaje de Fanon (2009, p. 42): "Hay una zona de no-ser, una región extraordinariamente estéril y árida, una rampa esencialmente despojada, desde la que puede nacer un auténtico surgimiento. En la mayoría de los casos, el negro no ha tenido la suerte de hacer esa bajada a los verdaderos Infiernos". 
fenómeno emergió hace más de quinientos años como una estrategia que se globaliza, y que va segregando cada vez más a punta de la aniquilación de todo aquello que se le oponga. Por lo tanto, el proyecto de muerte es parte esencial de la colonialidad ${ }^{2}$.

\section{Sobre la línea de color y la topología del ser: aportes de la razón negra a la com- prensión del problema ontológico de la colonialidad}

Este asunto de la operatividad del poder colonial en un sentido ontológico se puede ilustrar de manera más profunda a partir de los trabajos de William Du Bois, entre otros importantes pensadores de la diáspora africana ${ }^{3}$, quienes han proporcionado, en sus esfuerzos intelectuales y luchas políticas, un arsenal de herramientas para contribuir el campo que aquí reconocemos como la dimensión ontológica de la colonialidad. Sobre todo, por la sensibilidad y la relación particular que se manifiesta en su experiencia vivida con el hecho colonial racial. Ciertamente habría que pensar no sólo la posibilidad de encontrar recursos en los trabajos de estos y otros tantos autores que han plasmado diferentes elementos del ser-en-el-mundo del colonizado, del horizonte de comprensión que funda la experiencia vivida del sujeto colonizado, racializado. De igual manera, su pertinencia y potencia analítica radica en el establecimiento de límites a la filosofía moderna-occidente ${ }^{4}$. Se asume aquí el concepto de 'limite', como una incapacidad manifiesta de Europa y su herencia filosófica, para comprender otras realidades a partir de sus propias tradiciones, es decir, más allá del sesgo eurocéntrico presente en importantes pensadores como Espinosa, Kant o Hegel. Asimismo, consideramos el límite como una insolvencia para reconocer el sesgo racista en la comprensión de fenómenos sociales y ambientales.

2. Los indígenas de la Nasa, del Cauca en Colombia, utilizan el término de "proyecto de muerte", para referirse al panorama que viven frente a la devastación del mundo colonial. Puntualmente señalan: "el proyecto de muerte es la enfermedad del egoísmo que se vuelve odio, guerra, mentiras, propaganda, confusión, corrupción y malos gobiernos". A esto añaden que dicho fenómeno viene de la mano con las dinámicas desarrollistas y es estratégico. Por un lado, se presenta en forma de políticas de Estado, que induce a estos pueblos a librar una guerra que no es suya. Por otro lado, los despoja de su territorio, que destruye su cultura, que los obliga a asumir roles como informantes o a hacer parte activa de grupos armados (legales o ilegales), que entrega la naturaleza a empresas multinacionales para que se enriquezcan con la miseria de los pueblos. Cf. Asociación de Cabildos Indígenas del Norte del Cauca. (2004).

3. Por mencionar algunos: Frantz Fanón, Aimé Césaire, Angela Davis, Édouard Glissant, Achille Mbembe, Emmanuel Chukwudi Eze, etc., Para tener síntesis de estos debates se sugiere revisar: Cf. (Ondó, 2006). Cf (Bell, 2004). Cf. (Eze, 1997). Cf. (Afolayan \& Falola, 2017).

4. Sobre esto se pueden ver textos como: Mignolo (2017) y Maldonado-Torres (2012). 
Dicho proceso de violencia, que supone el proyecto de muerte de la colonialidad y que permea en la zona más íntima del ser, es una de las consecuencias de la operatividad de la diferencia ontológica colonial sobre las personas que son objeto de la dinámica colonialista, al clasificarlas, ordenarlas, asignarles identidades y roles dentro de la sociedad como "lugares naturales", incluso restringiendo la vida y generando muerte. Como se puede entrever, este mecanismo de la diferencia ontológica colonial evidenciaría, de entrada, dos caracteres: por un lado, aparece como una frontera que funciona tanto como instrumento de identificación, pero a la vez de exclusión y de distanciamiento, propia de la naturaleza antagónica de este poder. De otro lado, como un sistema de situalidad, de posicionamiento jerárquico del ser.

Con respecto a lo primero, hay que destacar que ha sido Du Bois quien ha pregonado que el principal problema del siglo XX es justamente la línea de color (2007, p.15). Para este autor, la línea de color existe en un plano exterior, pero no independiente, de la experiencia vivida del sujeto colonizado, para definir las oportunidades y acceso (o mejor la dificultad del mismo) a instituciones de índole educativa, política o económica. Pero al mismo tiempo, se interioriza en la mente y los cuerpos de los sujetos en una suerte de velo. Dicho velo, existe en las mentes de las personas y obliga a los blancos a estructurar la sociedad de acuerdo con una lógica racista: construir y vigilar a lo largo de la línea de color.

$\mathrm{Du}$ Bois argumenta que el velo evita que los blancos vean a los negros como ciudadanos (en su caso estadounidenses) y los traten como seres humanos. El velo, a su vez, impide que las personas negras se vean a sí mismas como realmente son, fuera de la visión negativa de la negrura creada por el racismo. De esta forma el negro ( $e l$ problema del negro) es una como un hijo nacido con un velo y dotado con una segunda vista (second-sight) en el mundo, un mundo que no le atribuye una verdadera auto conciencia, sino sólo le deja verse a través de la revelación del otro mundo (Du Bois, 2007, p. 8).

Este recurso de la "línea de color" es muy potente para explicar la operación antagónica en que funciona la diferencia ontológica colonial, ciertamente en la medida en que a través de la interiorización del velo se gesta una doble conciencia, se constituye a la vez lo que el sujeto (negro) "es", aquello que lo define en un estado ficcional:

Es una sensación peculiar, esta conciencia doble, este sentido de siempre verse a uno mismo a través de los ojos de otro, de medir la propia alma con el metro de un mundo que le mira con jocoso desprecio y lástima. Uno siempre siente su duplicidad, --un americano, un negro, dos almas, dos pensamientos, dos esfuerzos irreconciliables; dos ideas en combate en un cuerpo oscuro, cuya fuerza inflexible sólo se mantiene estando violentamente separadas (Du Bois, 2007, p. 8). 
Este devenir en una condición sub-ontológica, es decir, estar al interior de la frontera que se define por la línea de color y que se hace invisible a partir del velo constitutivo, manifiesta la centralidad, de aspectos concernientes a la raza en la analítica del poder colonial. Como dijera Fanón (1994, p. 31): "la colonización introduce en el ser un ritmo propio, aportado por los nuevos hombres, nuevo lenguaje, nueva humanidad". Por ello, autores como Calvin Warren afirman que la cuestión del Negro y la cuestión del ser están intrínsecamente relacionadas (Warren, 2018). A partir de esto se puede entender que la palabra 'negro' no remite solamente a la condición que se les impuso a las personas de origen africano durante el primer capitalismo (depredaciones de distinta índole, desposesión de todo poder de autodeterminación $y$, sobre todo, del futuro y del tiempo), sino que se asume como una nueva norma de existencia y su propagación al resto del planeta, lo que llamamos el devenir-negro del mundo (Mbembe, 2013).

Si se piensa entonces que ser pobre es difícil y que por ello la lucha relevante y preponderante debe ser la lucha de clases, entonces no se ha llegado a una adecuada comprensión de las implicaciones del poder colonial. Es aún peor "ser de una raza pobre", donde, como dice Du Bois, el negro siente el peso de la propia ignorancia, no solamente de las letras, sino también de la vida, de los negocios, de las humanidades; la pereza y la dejadez y la ruindad acumulada por décadas y siglos encadenaba sus manos y pies (Du Bois, 2007, p. 12).

Ahora bien, como segundo carácter la diferencia ontológica colonial en tanto sistema de situalidad manifiesta, lo que Maldonado-Torres (2006) denomina como la topología del ser. Si con el anterior carácter se establecía una relación entre la raza y la conciencia del sujeto colonizado, aquí se establece una relación entre la raza y el espacio, donde se despliega la experiencia. Esto ya se había sugerido en líneas anteriores. No obstante, vale la pena acentuar este carácter teniendo en cuenta que más allá de las justificaciones biológicas del racismo, o justificaciones basadas en las diferencias de cultura o modales, se puede encontrar en algunas tendencias influyentes en el pensamiento occidental una justificación ontológica y epistemológica más sutil:

... la fusión de raza y espacio está detrás de concepciones imperiales y militares de espacialidad que tienden a dar un nuevo significado al relato clásico de Agustín de las ciudades terrenales y celestiales: la diferencia entre la Ciudad de Dios y la Ciudad Terrenal de Hombres es traducido en la división entre las ciudades imperiales de los dioses humanos y las ciudades de los condenados. Desafortunadamente, la búsqueda de raíces en Europa y la geopolítica racista a menudo van de la mano (Maldonado-Torres, 2004, p. 51). 
De esta forma, la diferencia ontológica colonial, en tanto topología del ser, se trata también de una cartografía del poder colonial, que no sólo se obvia (oculta) en los ejercicios filosóficos, sino que además se legitima. Por ello, agrega Maldonado-Torres mientras, por ejemplo, Heidegger intenta encontrar raíces en la tierra, y Lévinas fundamenta la filosofía en dos ciudades (Atenas y Jerusalén), Fanon abre un camino de reflexión que toma las diferencias coloniales como un punto de partida para el pensamiento crítico. Una descripción crítica de la topología europea del ser y su geopolítica del conocimiento debería llevar a hacer visible lo que hasta ahora ha permanecido invisible o marginal y descubrir cómo funcionan las categorías filosóficas en función de la continuidad del poder colonial (Maldonado-Torres, 2004, pp. 51-52). De allí justo radica la importancia de una analítica de la dimensión ontológica de la colonialidad y, por tanto, de la diferencia ontológica colonial, como espacio de lucha y de desplazamiento de aquello que se ha naturalizado en los sujetos colonizados.

Por último, habría que agregar que esta topología evidencia justamente la actitud imperial. Se hace latente la correlación entre la constitución de un meta-saber trascendental y un ideal racial. El punto es que los sujetos asumen un lugar, no solo a nivel de la organización internacional del trabajo y de roles sociales (como lo plantea Quijano), sino también se hallan dentro de una lógica totalizante, estática, sin matices, impuesta, de la cual no parece haber escapatoria, que Glissant (1997, pp. 22-23) denomina caos-mundo. Un mundo heterogenizado, marcado por el escepticismo y la actitud imperial.

Y ciertamente no parece haber escapatoria porque el sujeto colonizado (el damné) no tiene posibilidad de resistencia, no solo política, sino también ontológica. Lo cual, a nuestro decir, obedece a la implantación de mecanismos como la raza y el género que se instalan en el ámbito significativo de la experiencia del sujeto colonizado, es decir, en condiciones que genera criterios de espacialidad y temporalidad para situar determinadas comunidades en posiciones de superioridad y otras en condiciones de inferioridad ${ }^{5}$.

5. En este punto vale la pena hacer presente nuevamente las palabras de Fanón (2009) "El negro no tiene resistencia ontológica frente a los ojos del blanco. Los negros, de un día para otro, han tenido dos sistemas de referencia en relación a los cuales han debido situarse. Su metafísica o, por decirlo de manera menos pretenciosa, sus costumbres y las instancias a las que éstas remitían, fueron abolidas porque se contradecían con una civilización que ellos ignoraban y que se les imponía" (p. 112). 


\section{Operatividad del poder colonial en el marco del sexismo: a propósito de la colo- nialidad del género}

En sintonía a lo anterior, el otro eje clave en la comprensión de la dimensión ontológica de la colonialidad es el género. De la mano de María Lugones conectamos el abordaje con el marco del feminismo decolonial ${ }^{6}$. Para esta autora el género se concibe como una imposición colonial que abre un escenario de despliegue de la colonialidad desde el inicio mismo de la modernidad, lo cual se reconoce como la colonialidad del género. Según Lugones (2011):

El pensar acerca de la colonialidad del género nos permite pensar en seres históricos que sólo son comprendidos como oprimidos de un modo simplista y unilateral. Puesto que no existen seres que sean mujeres colonizadas... Tales seres son, como he sugerido, sólo parcialmente comprendidos como oprimidos, en la medida en que son construidos a través de la colonialidad del género (p. 109).

Señalar que el racismo y sexismo como efectos del poder colonial y como mecanismo de reproducción del mismo, se encuentran inherentemente ligados en la misma matriz, permite reconocer, como lo hace Lugones, que la raza no es ni más mítica ni más ficticia que el género - ambos son ficciones poderosas (Lugones, 2008, p. 94). Pero son ciertamente eso, ficciones. Esta apuesta reconoce aquellos "seres históricos", a los cuales hace alusión Lugones en la anterior cita. Aún más, reconoce que estos seres "no son" más que en la medida en que "son" construidos ontológicamente a través de diferentes mecanismos de poder colonial, como el que nos ocupa en este caso, a saber, la colonialidad del género y mediante la diferencia ontológica colonial. Este proceso de "construcción de seres", o como también lo denomina Lugones (2011, p. 111) "subjetificación" conlleva a la adopción, naturalización de, entre otras cosas, la dicotomía entre hombres y mujeres como una construcción normativa de lo social - una señal de civilización, ciudadanía y membrecía en la sociedad civil.

6. Existe importante literatura que aporta elementos de sobremanera ilustrativos y relevantes en materia del feminismo decolonial. Sería pertinente en otro trabajo hacer una lectura mucho más sistemática de los aportes del feminismo decolonial a la comprensión del problema de la operatividad del poder colonial en clave ontológica. Por razones metodológicas y de extensión hemos decidido seguir más de cerca los planteamientos de María Lugones. No obstante, vale la pena señalar algunos trabajos relevantes en esta línea. Solo por mencionar algunas autoras: Espinosa (2009; 2014), Barroso (2014); Curiel (2007); Segato (2003; 2012); Rivera Cusicanqui (2010; 2015); Federici (2014; 2018) Flórez-Flórez (2007) Gómez-Correal (2014 o 2011); entre otras autoras que han hecho especiales contribuciones a este campo de estudios. 
Esta dicotomía promueve diferentes representaciones simbólicas (discursivas) en el ámbito de la sexualidad, pero aún más y como todo mecanismo de poder colonial, genera prácticas de exclusión, segregación e invisibilización. De allí que se pueda afirmar que la dicotomía de género (representación discursiva) funciona normativamente en la construcción de lo social y en la construcción de los procesos coloniales de subjetificación opresiva, por ejemplo, legitimando, o al menos haciendo invisibles o naturales las prácticas de violencia sexistas (p. 112).

Para ilustrar lo anterior, se puede ver en el panorama social que el sexismo es un tema del que no se habla en espacios de incidencia política, y cuando se habla, es minimizado, despreciado o ridiculizado ${ }^{7}$. Se pueden encontrar constantes casos en los que las mujeres presentan argumentos contundentes, y por qué no, pasionales, emocionales con el sentimiento que produce la injusticia, a lo cual de inmediato se ridiculiza o se justifica, simplemente porque "es mujer" y "seguramente se merecía lo que le sucedió". En este plano son marginadas, ridiculizadas, infantilizadas, dando lugar a un proceso de subjetificación en el que éstas deben ser construidas como sumisas, silenciosas, leales y buenas trabajadoras.

En este punto me parece pertinente recordar las palabras de Lugones (2011) sobre la colonialidad vista desde la perspectiva de la colonialidad del género:

Ver la colonialidad es ver la poderosa reducción de seres humanos a animales, inferiores por naturaleza, en una comprensión esquizoide de la realidad que dicotomiza lo humano de la naturaleza, lo humano de lo no humano, y que impone una ontología y cosmología que, en su poder y su constitución, no permite toda la humanidad, toda posibilidad de comprensión, toda posibilidad de comunicación humana, a los seres deshumanizados (Lugones, 2011, p. 114).

Leer esta problemática desde el nivel ontológico lleva a complejizar un poco más la tarea de hacer visibles los mecanismos de poder colonial. Por un lado, se complejiza, porque lleva a reconocer que no es suficiente visualizar de manera aislada cada línea de fuerza (raza y género), sin manifestar las repercusiones y afectaciones reciprocas que estas tienen entre sí. Así por ejemplo, la idea de Lugones (2008) de investigar la intersección de raza, clase, género y sexualidad para entender la "preocupante indiferencia" que los hombres muestran hacia las violencias que sistemáticamente se infringen sobre las mujeres de color (Lugones, 2008, p. 75), se constituye en un claro ejercicio de lo que aquí hemos venido dilucidando y, por ende, en un soporte importante para avanzar en la comprensión del nivel ontológico de la colonialidad ${ }^{8}$.

7. Para el caso colombiano este es un asunto que ha quedado reducido a la etiqueta de "feminismo trasnochado".

8. En este punto vale la pena destacar el valor teórico y metodológico del denominado "giro interseccional" en la teoría feminista. Sobre este asunto Viveros (2016) ha realizado una genealogía de los 
De otro lado, se trata de entender que conectar la perspectiva de género con el cuestionamiento de la forma cómo opera el poder colonial no es solamente un asunto caprichoso o de simple figuración. Es una tarea apremiante de cuestionar los privilegios "patriarcales", que se han naturalizado, incluso de alguna manera en la exposición de autores (hombres) decoloniales. De allí, aún más cala la gesta de Lugones, por entender tal actitud displicente que los hombres evidencian (evidenciamos) ${ }^{9}$ hacia las diferentes formas de violencia contra las mujeres ${ }^{10}$.

En esta dirección y en referencia al trabajo de Quijano, Lugones (2008) hace algunas menciones:

En el patrón de Quijano, el género parece estar contenido dentro de la organización de aquel «ámbito básico de la existencia» que Quijano llama «sexo, sus recursos y productos». Es decir, dentro de su marco, existe una descripción de género que no se coloca bajo interrogación y que es demasiado estrecha e hiper-biologizada ya que presupone el dimorfismo sexual, la heterosexualidad, la distribución patriarcal del poder y otras presuposiciones de este tipo... Podemos ver que el alcance de la colonialidad del género en el análisis de Quijano es demasiado limitado. Para definir el alcance del género, Quijano asume la mayor parte de lo prescripto por los términos del lado visible/claro hegemónico del sistema de género colonial/moderno. He tomado un camino que me ha llevado afuera del modelo de Quijano de la colonialidad del género para revelar lo que el modelo oculta, o que no nos permite considerar, en el alcance mismo del sistema de género del capitalismo global eurocentrado (pp. 82-88).

\footnotetext{
enfoques interseccionales, incluyendo los aportes del pensamiento feminista producido en distintos contextos históricos y geopolíticos. De igual forma, ha identificado algunas de las grandes líneas del debate sobre la interseccionalidad con el fin de mostrar su alcance y sus límites, ligados en gran parte a su amplia difusión.

9. Hago esta acentuación porque de alguna forma mi condición privilegiada de hombre me ha permitido que pueda llevar a cabo (tal vez de alguna forma "más fácil') este tipo de prácticas académicas. De allí que la decolonialidad y en especial en el ámbito de la colonialidad del género, deba asumirse como una cuestión de desprendimiento de privilegios. A este respecto, recomendamos ver la entrevista realizada a Suárez-Krabbe (2017).

10. En palabras de Lugones (2008): "Esta indiferencia es insidiosa porque impone barreras impasables en nuestras luchas como mujeres de color por nuestra propia integridad, autodeterminación, la médula misma de las luchas por la liberación de nuestras comunidades. Esta indiferencia se halla tanto al nivel de la vida cotidiana como al nivel del teorizar la opresión y la liberación. La indiferencia no está provocada solamente por la separación categorial de raza, género, clase y sexualidad, separación que no nos deja ver la violencia claramente. No se trata solamente de una cuestión de ceguera epistemológica cuyo origen se radica en una separación categorial” (p. 76).
} 
Lo anterior permite evidenciar que no es suficiente una lectura crítica de los efectos de la empresa colonial sobre realidades como las de América Latina, para lograr exteriorizar la profundidad de dicha matriz constituyente de poder y su modo de operación. La colonialidad está continuamente reorganizándose y encarnando mecanismos diversos para su propia reproducción. Por ello, se justifica aún más la comprensión ontológica de los mecanismos que posibilitan dicha dinámica (colonialidad del ser-colonialidad del género), asumiendo los retos que ello implica, tales como entrar en diálogo con otras perspectivas (feminismo decolonial, interculturalidad), descentrar el privilegio de la academia en la producción de conocimientos, volcarse a la acción social, entre otros.

Esta misma situación la hace explícita Silvia Federici, con su aproximación crítica al marxismo. En el centro de su crítica, esta autora argumenta que el análisis del autor alemán sobre el capitalismo se ha visto lastrado por su incapacidad de concebir el trabajo productor de valor, de una forma diferente que no sea la producción de mercancías y su consecuente ceguera sobre la importancia del trabajo no asalariado de las mujeres en el proceso de acumulación capitalista. En otros términos, las limitaciones que trae la perspectiva marxista respecto a la cuestión de la "reproducción" vista desde una perspectiva provincial y no planetaria. Esto pues no es capaz de ver el trabajo más allá de los términos de mercancía e invisibiliza el valor del trabajo doméstico en las dinámicas económicas (Federici, 2010). Obviar este trabajo, como lo señala Federici, limitó la comprensión de Marx del verdadero alcance de la explotación capitalista del trabajo y de la función que el salario desempeña en la creación de divisiones dentro de la clase trabajadora, comenzando por la relación entre mujeres y hombres (Federeci, 2018).

En lugar de apreciar el valor del trabajo doméstico o de las diversas luchas colectivas, el marxismo ha preferido legitimar la necesidad del trabajo asalariado y potenciar la lucha de la clase trabajadora (proletariado) como única lucha auténtica en, lo que para Marx sería, el devenir histórico y necesario del capitalismo. Adicionalmente, hay que anotar una tendencia existente en el marxismo a concebir al capitalismo como un "sistema" autorregulado, expansivo, victorioso, con racionalidad propia, totalizante, omnipresente, dinámico, generativo, autosuficiente, maleable, axiomático, poderoso, auto-organizado, homogéneo, resistente a la crisis, etc. (Federeci, 2018).

Evidentemente Marx, y posteriormente sus seguidores, no dimensionaron la estrecha relación existente entre asuntos como la colonialidad, el capitalismo el racismo y el patriarcado. Operativizar el poder colonial en este sentido significaría desconocer las otras luchas, más allá de la lucha de clases, que se inscriben en campos como el de la raza y el género, que combatirían no sólo el capitalismo, sino a la colonialidad que ha hecho posible este juego de relaciones de dominación. Es decir, dinamizar la colonialidad a través de la supremacía de una categoría como la de lucha de clases, 
implicaría una "totalización" de las relaciones de dominación capitalista, bajo el espectro otorgado por la categoría de "clase social", una "separación" de los diferentes ámbitos de la vida histórico-social (permeados por criterios patriarcales y racistas), de los diversos agentes de acción política. Revertir esta situación exigiría una lectura microscopia de las interrelaciones existentes entre la clase, la raza, el género y la sexualidad, en orden del mantenimiento de la empresa colonial-capitalista que legitima el desarrollo. Sobre esto, el feminismo decolonial nos ha dado grandes lecciones de las cuales podemos aprender para avanzar hacia la decolonización de estas relaciones de poder. De esta manera, el trabajo de Ángela Davis, el de María Lugones, la misma Federici y de otras muchas feministas decoloniales que hemos mencionado, se ha concentrado en investigar la intersección de raza, clase, género y sexualidad como un aporte fundamental e imperante en el análisis de la operatividad del poder colonial, sobre todo en el marco de su despliegue ontológico.

\section{A manera de conclusión}

Varios elementos quedan por acentuar y seguramente por desarrollar con mayor profundidad a propósito de la lectura ontológica que proponemos de las prácticas sexistas y en general de la colonialidad. No obstante, es importante seguir trabajando en el reconocimiento de esta problemática, pues como se ha insistido a lo largo del documento, el hecho de no reconocer los elementos ontológicos en la comprensión de la operatividad del poder colonial en el plano de las prácticas sexistas y racistas es continuar con la reproducción de la lógica colonial. La raza y el género no son asuntos accesorios o accidentales en el abordaje de los efectos del poder colonial. Hacen parte del núcleo central de la matriz de poder que llamamos colonialidad, que continúa teniendo efectos inmediatos y materiales en realidades que han cargado con el yugo colonialista.

La idea de raza 'de-vela' asuntos fundamentales de la experiencia vivida del sujeto colonizado. Abre la posibilidad de identificar mecanismos, como el de la línea de color, a partir de los cuales se tiende a inferiorizar, violentar o a infantilizar al colonizado. La huella racista en el plano del ser es uno de los grandes retos de la filosofía de nuestros tiempos, pues como hemos dicho no es un asunto exclusivo del problema del negro.

En materia de la cuestión del género al interior de la analítica de la colonialidad, se puede advertir que el aporte de autoras como María Lugones, se constituyen en una contribución fundamental para avanzar en dicha tarea de desmitificación y desentrañamiento ontológico. Si bien Lugones no señala explícitamente o atribuye directamente la lógica del poder colonial con relación al plano del género y del ser a la manera cómo opera la diferencia ontológica colonial (pues en sus trabajos la categoría utilizada es la de "diferencia colonial"), esto no significa que no desarrolle una lectura ontológica del problema. En sus trabajos evidencia de sobremanera y con argumen- 
tos claros el funcionamiento del poder colonial a nivel ontológico, que abre puertas y ofrece pistas importantes para continuar con el proyecto decolonizador.

Adicionalmente, se debe destacar en lo concerniente a la colonialidad del género que la producción de la muerte se esconde detrás de la diferencia ontológica colonial en el marco violencia de las prácticas sexistas. Entendemos aquí la muerte no sólo desde el punto de vista físico, sino también como la muerte civil, social, política de aquellos seres situados en escalas de inferioridad ((Losada, 2014; Suárez-Krabbe, 2016). En este sentido, la diferencia ontológica colonial es una forma de producir la muerte e impedir que los sujetos "racializados" y "sexualizados" tengan condiciones para participar en la vida civil, social y política.

Este nivel de operatividad del poder colonial, que encuentra en la raza y el género sus mejores aliados, es invisibilizado, ocultado por el mismo sujeto colonial, puesto que afecta directamente aquello que hace parte de su intimidad, aquellas ficciones a partir de las cuales se reconoce y bajo las que desarrolla sus prácticas cotidianas como parte de lo que "es".

Por ello, y en sintonía con Lugones, no creemos que sea una simple cuestión de pasividad. Es, por el contrario, una estrategia muy activa de la lógica colonial que motiva, por ejemplo, políticas de gobiernos de derecha a pasar por alto las consecuencias del racismo y el sexismo, manifestando su inexistencia en tiempos actuales, desconociendo las conexiones con la violencia intrafamiliar, los feminicidios, desigualdades en escalas salariales, acceso a educación, etc.

De otro lado, queremos alertar sobre la necesidad de entender el proceso de universalización y legitimación de prácticas sexistas que con su lógica de naturalización e inferiorización dificultan el diálogo, o al menos el reconocimiento de iguales condiciones de poder, hacia la búsqueda de alternativas al orden excluyente y de dominación auspiciado por la colonialidad. Lo cual no significa que no se puedan pensar y hacer proyectos de transformación de decolonialidad, que de entrada ya existen, están latentes y cada vez se hacen más poderosos. En este sentido, por ejemplo, se puede leer las experiencias de mujeres afroamericanas, chicanas y lesbianas en Estados Unidos, como las luchas de las mujeres de las comunidades originarias y afrodescendientes de Abya Yala, así como el trabajo zapatista en México, entre otras, que invitan y que alientan a continuar trabajando en esta labor. En Colombia se han tejido diversas redes de resistencias en sectores estratégicos como el pacífico, la región amazónica, los Montes de María en la región caribe, en torno a diversas situaciones derivadas del legado colonialista. De allí que otra tarea pendiente sea justamente la de visibilizar estas experiencias de cambio, dialogar con ellas y nutrirse de su potencial emancipador.

Finalmente, no queremos dejar de señalar que, si bien pensar en términos ontológicos pareciese situar la discusión en un plano abstracto con poca atención la vida fáctica y a la praxis cotidiana, lo que abre es justamente la posibilidad de repensar 
las condiciones materiales en las que se insertan nuevas y constantes dinámicas de poder (reales) de violencia y de muerte derivadas de la legitimidad y naturalización de la diferencia ontológica colonial. Así mismo, permite reconocer el carácter emancipador y decolonizador de las prácticas cotidianas de contornos ontológicos de grupos históricamente oprimidos. Esto pues, hay que propender por la formulación de contra-poéticas como lo dijera Glissant (1981), que además de velar por una desobediencia epistémica (Mignolo, 2015), también apunten a la desobediencia ontológica, nuevos sentires, nuevos vivires, nuevas formas de re-existencia. Se trata en definitiva de combatir la política de la muerte, de quitarle el velo al poder colonial, se trata de vivir bien.

\section{Referencias}

Afolayan, Adeshina \& Toyin Falola (2017).The Palgrave Handbook of African Philosophy. Palgrave Macmillan, New York, 2017.

Asociación de Cabildos Indígenas del Norte del Cauca (2004). Propuesta Política y de Acción de los Pueblos Indígenas. Recueprado de http://anterior.nasaacin.org/ index.php/propuesta-politica-de-los-pueblos.

Barroso, José María (2014). "Feminismo decolonial: una ruptura con la visión hegemónica eurocéntrica, racista y burguesa. Entrevista con Yuderkys Espinosa Miños”o. Iberoamérica Social: revista-red de estudios sociales (III), pp. 22 - 33. Recuperado de http://iberoamericasocial.com/feminismo-decolonial-una-rupturacon-la-vision-hegemonica-eurocentrica-racista-yburguesa.

Bell, Richard (2004). Understanding African philosophy: A cross-cultural approach to classical and contemporary issues. Routledge.

Castro-Gómez, Santiago (1996). Crítica de la razón latinoamericana. Barcelona: Puvill Libros.

Castro-Gómez, Santiago (2005). La hybris del punto cero: ciencia, raza e Ilustración en la Nueva Granada (1750-1816). Bogotá: Centro Editorial Pontificia Universidad Javeriana.

Castro-Gómez, Santiago (2007). Michel foucault y la colonialidad del poder. Tabula Rasa, 6: 153-172.

Castro-Gómez, Santiago (2009). Tejidos Oníricos. Movilidad, capitalismo y biopolítica en Bogotá (1910-1930) Bogotá: Centro Editorial Pontificia Universidad Javeriana/ Instituto Pensar.

Curiel, Ochy (2007). Crítica poscolonial desde las prácticas políticas del feminismo antirracista. Nómadas (Col), (26).

Du Bois, W. E. B. (2007). The Souls of Black Folk. Oxford University Press Inc. 
Dussel, Enrique (1992). 1492: El encubrimiento del otro. El origen del mito de la modernidad. Bogotá: Anthropos

Dussel, Enrique (1996). Filosofía de la Liberación. Bogotá, Editorial Nueva América.

Dussel, Enrique (1999). Más allá del eurocentrismo: el sistema mundo y los límites de la modernidad. En: S. Castro Gómez, O. Guardiola Rivera y C. Millan De Benavides (Ed.) Pensar (en) los intersticios. Teoría y práctica de la crítica poscolonial. Bogotá: CEJA,.

Dussel, Enrique (2005). Europa, modernidad y eurocentrismo. En: LANDER, Edgardo. La colonialidad del saber: eurocentrismo y ciencias sociales. Perspectivas latinoamericanas. La Habana: Editorial de ciencias sociales.

Escobar, Arturo (1996). La invención del tercer mundo. Santafé de Bogotá: Norma.

Escobar, Arturo (1999). El final del salvaje: Naturaleza, cultura y politica en la antropología contemporánea. Bogotá: CEREC, ICAN.

Espinosa, Yuderkys (2009). Etnocentrismo y colonialidad en los feminismos latinoamericanos: complicidades y consolidación de las hegemonías feministas en el espacio transnacional. Revista venezolana de estudios de la mujer, 14(33): 37-54.

Espinosa, Yuderkys (2014). Una crítica descolonial a la epistemología feminista crítica. El Cotidiano, 29(184): 7-12.

Eze, Emmanuel Chukwudi (Ed.) (1997). Postcolonial African philosophy: A critical reader. Cambridge: Blackwell Publishers.

Fanon, Frantz (2009). Piel negra, máscaras blancas. Madrid: Ediciones Akal.

Fanón, Frantz (1994). Los condenados de la tierra. México: FCE.

Federici, Silvia (2010). Calibán y la bruja. Mujeres, cuerpo y acumulación originaria. Madrid: Traficantes de sueños.

Federici, Silvia (2014). La inacabada revolución feminista. Mujeres, reproducción social y lucha por lo común. Bogotá: Ediciones desde abajo.

Federici, Silvia (2018). El patriarcado del salario. Críticas feministas al marxismo. Madrid: Traficantes de sueños.

Flórez-Flórez, Juliana (2007). "Lectura no eurocéntrica de los movimientos sociales latinoamericanos. Las claves analíticas del proyecto modernidad/colonialidad". En: S. Castro-Gómez y R. Grosfoguel (eds.) (2007). El giro decolonial. Reflexiones para una diversidad epistémica más allá del capitalismo global (pp. 243-266). Siglo del Hombre Editores, Universidad Central y Pontificia Universidad Javeriana, Bogotá.

Glissant, Edouard (1981). Le discours antillais. Paris, Editions de Seuil.

Glissant, Edouard. (1997). Traité du tout-monde. Éditions Gallimard. 
Gómez-Correal, Diana (2011). Feminismo y modernidad/colonialidad: entre retos de mundos posibles y otras palabras. En: Y. Espinosa, D. Gómez \& K. Ochoa (Eds.). Tejiendo de otro modo: Feminismo, epistemología y apuestas descoloniales en Abya Yala. Popayan, Universidad del Cauca.

Gordon, Lewis (1999). Bad Faith and Antiblack Racism. New York: Humanity Books Grosfoguel, Ramón (2003). Colonial Subjects. Berkeley: University of California Press. Grosfoguel, Ramón (2005). The Implications of Subaltern Epistemologies for Global Capitalism: Transmodernity, Border Thinking and Global Coloniality. En Richard P. Appelbaum and William I. Robinson (eds.). Critical Globalization Studies. New York /London: Routledge.

Grosfoguel, Ramón (2006). La descolonización de la economía política y los estudios postcoloniales: Transmodernidad, pensamiento fronterizo y colonialidad global. Tabula Rasa, 4, 17-46.

Lander, Edgardo (ed.). (2005). La colonialidad del saber: eurocentrismo y ciencias sociales. Buenos Aires: CLACSO.

Losada, Jhon (2014). Ontología y poder colonial: Claves analíticas a propósito de la Colonialidad del Ser. Bogotá: Editorial Bonaventuriana.

Lugones, María (2008). Colonialidad y Género. Tabula Rasa, 9, 73-101.

Lugones, María (2011). Hacia un feminismo descolonial. Revista La manzana de la discordia, 6(2): 105-117.

Maldonado-Torres, Nelson (2003). Thinking From the Limitis of Being: Levinas, Fanon, Dussel and the Cry of ethical revolt. New York: Routledge.

Maldonado-Torres, Nelson (2004). The topology of being and the geopolitics of knowledge: Modernity, empire, coloniality. City, 8 (1): 51-78.

Maldonado-Torres, Nelson (2006). La topología del ser y la geopolítica del saber: modernidad, imperio, colonialidad. En F. Schiwy y N. Maldonado-Torres (Eds.), (Des) Colonialidad del ser y del saber (videos indígenas y los límites coloniales de la izquierda) en Bolivia. Argentina: Ediciones El Signo.

Maldonado-Torres, Nelson (2007). Sobre la colonialidad del ser: contribuciones al desarrollo de un concepto. En: S. Castro-Gómez y R. Grosfoguel (eds.), El giro decolonial: reflexiones para una diversidad epistémica más allá del capitalismo global (pp. 127-167). Bogotá: Universidad Javeriana-Instituto Pensar, Universidad Central-IESCO y Siglo del Hombre Editores.

Maldonado-Torres, Nelson (2012). Thinking at the limits of philosophy and doing philosophy elsewhere. From Philosophy to Decolonial Thinking. En: G. Yancy (Ed.). Reframing the Practice of Philosophy: Bodies of Color, Bodies of Knowledge. SUNY Press. 
Mbembe, Achille (2013). Critique de la raison négre. Paris, La Découverte.

Mignolo, Walter (1995). The Darker Side of the Renaissance, Literacy, Territoriality and Colonization. Ann Arbor: University of Michigan Press.

Mignolo, Walter (2000). Local Histories/Global Designs. Coloniality, Subaltern Knowledges, and Border Thinking. Princeton, NJ: Princeton University Press.

Mignolo, Walter (2005). The Idea of Latin America. Oxford: Blackwell.

Mignolo, Walter (2015). La désobbéissance épistemique. Rhétorique de la modernité, logique de la colonialité et grammaire de la décolonialité. Bruxelles, Peter Lang.

Mignolo, Walter (2017). The Advent of Black Thinkers and the Limits of Continental Philosophy. En: A. Afolayan \& T. Falola. The Palgrave Handbook of African Philosophy. Palgrave Macmillan, New York.

Ondó, Eugenio (2006). Síntesis sistemática de la filosofía africana. Ediciones carena.

Quijano, Aníbal \& Immanuel Wallerstein (1992). La americanidad como concepto, o América en el moderno sistema mundial. Revista Internacional de Ciencias Sociales. América, 1492-1992, XLIV (4): 583 - 602.

Quijano, Anibal (1992). Colonialidad y modernidad-racionalidad. En H. Bonilla (Ed.), Los conquistados: 1492 y la población indígena de las Américas. Bogotá: Tercer Mundo-Flacso-Libri Mundi.

Quijano, Anibal (2000a). Colonialidad del poder, eurocentrismo y America latina. Colonialidad del Saber, Eurocentrismo y Ciencias Sociales, 201-246. CLACSOUNESCO 2000, Buenos Aires.

Quijano, Anibal (2000b). Colonialidad del Poder y Clasificacion Social, Festschrift for Immanuel Wallerstein, part I. Journal of World Systems Research, XI(2), summer/ fall.

Quijano, Anibal (2005). Colonialidad del poder, eurocentrismo y América Latina. En: La colonialidad del saber: eurocentrismo y ciencias sociales. LANDER, Edgardo (Comp.) Buenos Aires: CLACSO.

Quijano, Anibal (2007). Colonialidad del poder y clasificación social. En S. CastroGómez y R. Grosfoguel (eds.) (2007). El giro decolonial: reflexiones para una diversidad epistémica más allá del capitalismo global. Bogotá: Universidad Javeriana-Instituto Pensar, Universidad Central-IESCO y Siglo del Hombre Editores.

Rivera Cusicanqui, Silvia (2010). Ch’ixinakax utxiwa: una reflexión sobre prácticas y discursos descolonizadores - 1a ed. - Buenos Aires: Tinta Limón.

Rivera Cusicanqui, Silvia (2015). Sociología de la imagen: ensayos, - 1a ed. - Ciudad Autónoma de Buenos Aires: Tinta Limón. 
Segato, Rita (2003). Las estructuras elementales de la violencia: contrato y status en la etiología de la violencia (No. 334). Universidad de Brasília, Departamento de Antropologia.

Segato, Rita (2012). Gênero e colonialidade: em busca de chaves de leitura e de um vocabulário estratégico descolonial. e-cadernos ces, (18).

Suárez-Krabbe, Julia (2014). ¡El emperador está desnudo! Crítica de las prácticas hegemónicas de derechos humanos y desarrollo y alternativas desde el sur global. En: J. Losada, y J. Barrera (Eds.) Lecturas Criticas del Desarrollo: Perspectivas Latinoamericanas. Bogotá: Editorial Bonaventuriana.

Suárez-Krabbe, Julia (2016). Race, Rights and Rebels. Alternatives to Human Rights and Development from the Global South. New York, Rowman \& Littlefield international press.

Viveros, Mara (2016). La interseccionalidad: una aproximación situada a la dominación. Debate Feminista, 52, 1-17.

Warren, Calvin (2018). Ontological terror: Blackness, nihilism, and emancipation. Durham: Duke University Press.

Wynter, Sylvia (2004). Unsettling the coloniality of being/power/truth/freedom: Towards the human, after man, its overrepresentation--An argument. CR: The new centennial review, 3(3): 257-337.

\section{Sobre el autor}

Jhon Jairo Losada Cubillos es Magister en Filosofía Contemporánea y Doctorando en Filosofía y miembro del Centro de Filosofía del Derecho (CPDR) de la Universidad Católica de Lovaina. Correo Electrónico: jhon.losada@uclouvain.be. (D https:// orcid.org/0000-0001-8086-3516 


\title{
CUHSO
}

Fundada en 1984, la revista CUHSO es una de las publicaciones periódicas más antiguas en ciencias sociales y humanidades del sur de Chile. Con una periodicidad semestral, recibe todo el año trabajos inéditos de las distintas disciplinas de las ciencias sociales y las humanidades especializadas en el estudio y comprensión de la diversidad sociocultural, especialmente de las sociedades latinoamericanas y sus tensiones producto de la herencia colonial, la modernidad y la globalización. En este sentido, la revista valora tanto el rigor como la pluralidad teórica, epistemológica y metodológica de los trabajos.

\author{
EDITOR \\ Matthias Gloël \\ CoOrdinadora EDITORIAL \\ Claudia Campos Letelier \\ Corrector de ESTILO Y DiSEÑAdor \\ Ediciones Silsag \\ Traductor, CORRECTOR LENGUA INGLESA \\ Aurora Sambolin Santiago \\ SITIO WEB \\ cuhso.uct.cl \\ E-MAIL \\ cuhso@uct.cl
}

LICENCIA DE ESTE ARTÍCULO

Creative Commons Atribución Compartir Igual 4.0 Internacional 